\title{
Working in Spanish ICUs compared with UK ICUs
}

\author{
Alicia Martinez, RN, General Nurse, Virgin of Arrixaca Universitary Hospital, Murcia, Spain ( \\ Carmen Martinez, RN, Student Midwife, Canterbury, UK
}

A Martinez, Urb. Los Romeros C/B num 22, 30565 Las Torres de Cotillas (Murcia), Spain. e-mail: aliciamn@hotmail.com

\section{INTRODUCTION}

In 2000, we finished our nursing degrees at the University of Murcia, Spain. After this, we worked mainly in intensive care, firstly for six months in Spain, followed by four months in England. Our experience of working in both the English and Spanish National Health Services (NHS) has taught us two different working styles. In this article, we would like to highlight the differences and similarities we found between working in English and Spanish intensive care units (ICUs).

\section{NURSING ACTIVITIES}

In Spain, nursing activities are more technical, with decisions about diagnosis and treatment made only by doctors. Nurses can give their opinion, but they cannot change anything without the prior agreement of a doctor. In England, however, E- and Fgrade nurses are able to do arterial blood gases and change ventilator values for the benefit of the patient without having to ask a doctor first, though the doctor must be informed afterwards.

Both English and Spanish nursing activities involve the basic comfort care of the patient, including eye and mouth care, checking that everything is all right with the patient, informing the patient about any relevant changes, creating a therapeutic relationship with relatives, following the medical treatment, giving nursing care, giving medication (intravenous, subcutaneous and intramuscular). However, Spanish nurses activities also perform more technical procedures, such as taking venous and arterial blood (from the line or from the patient with the needle), cannulating veins and arteries, and inserting nasogastric catheters etc.

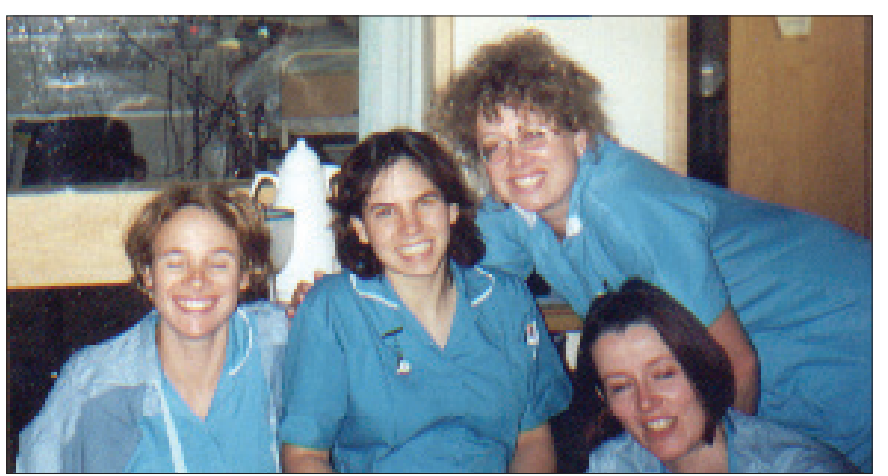

\section{NURSING CARE}

Nursing care is the basis of nursing in both countries. The patient is considered of the utmost importance in both countries, the person who needs someone to do the things he or she cannot do, or someone to help him or her to do them.

Interestingly, the attitudes of English and Spanish nurses towards both patients and relatives are different and may be a result of the different cultures. Thus, English nurses will smile at both patients and relatives, even if they do not like them (which is not sincere but needed by the family and patient). However, in Spain, it is permissible for the nurse to show a patient that one is tired or angry with him (which is sincere but not always polite).

In Spain, every nurse has to look after two patients (even if they are patients in a high-dependency unit (HDU) or ICU) and after three patients if they are from the cardiology ICU. In England, nurses work on a ratio of one nurse to each patient in intensive care and on a ratio of one nurse to two patients in HDU. However, in Spain, ICUs also have 'auxiliares' and 'celadores', as well as nurses. In the UK, only nurses can work in an ICU.

\section{Auxiliares}

Auxiliares are similar to English health care assistants. They wash or help the patient to wash themselves, bring them meals, stock up materials when needed, take a patient's temperature, check urine output and gastric contents, run the enteral nutrition pumps and give oral drugs under nurse supervision.

\section{Celadores}

Celadores act as 'carriers'. They take patients to the radiography department (accompanied by nurses who check that the patient remains stable) or theatre. They help nurses to change patient positions, sit patients up, supervise patient visits, and essentially carry out the physical work of nursing. This role does not exist in England. In England, health care assistants carry out similar work on the ward as done by Spanish auxiliares, but they do not do work in an ICU - only nurses can.

\section{Ward managers}

In Spain, ward managers look after the ward and not after the patients. Thus, every nurse cares for his patients on his own, giving the care that he or she thinks best, according to his or her own criteria. In England, the ward manager not only supervises the ward, but also supervises the care every nurse gives to his or her patient. 


\section{RELATIVES' VISITS}

Visiting by relatives is strictly controlled in Spanish ICUs. Visiting hours are from $12.30 \mathrm{pm}$ to $2 \mathrm{pm}$ and from $7.30 \mathrm{pm}$ to $9 \mathrm{pm}$. Doctors inform every family about any changes that have occurred in the last few hours. In England, as long as the nurse or the patient do not need privacy, e.g. to wash or perform a procedure, relatives can stay for the whole day.

\section{NURSING CARE Supernumerary nurse}

The first thing that surprised us when we began working in England was our position as supernumerary nurses for the first month of working.

In Spain, as soon as a nurse has finished his or her nursing degree, the nurse is supposed to know everything - or enough to look after patients, though of course this does not mean that Spanish nurses stop learning. When a nurse starts working, he or she has the same responsibilities and duties as the oldest and most experienced nurse in the ward. No first-month learning is allowed. Due to unemployment in Spain, newly qualified nurses get between one and three months' contract each year, so that spending one of the months as a supernumerary is not a choice.

\section{Nursing grades}

In Spain there are no D, E, F, or G grades. A nurse has the same responsibilities on the first day of nursing as he or she does 10 years later, unless of course the nurse has become a ward manager, who does not cope with patients but with papers.

\section{Handover procedure}

The handover of patients between nurses is different in Spain and the UK. In Spain, patient handover is usually carried out within 5-10 minutes at the patient's bedside, immediately to the next nurse who is going to look after the patient. Spanish nurses always take care of the same beds, so that a nurse will always look after the patients who stay in those beds.

In England, in the ICU in which we worked, the nurse-incharge gives the handover in the coffee room to all the nurses who will be working on next shift, so that everyone is aware of the situation in the ward and of all the patients. Then, the new nursein-charge assigns a patient to every nurse (not always the same patient nor the same beds). Following this, by the patient's bedside, the nurse of every patient gives a detailed handover to the new nurse who is going to stay with the patient for the next shift.

\section{Full-time nursing}

Working as a full-time nurse in Spain means working 35 hours per week (instead of 37.5 hours in England). In Spain, there is not the opportunity to work part-time. In England, nurses have the advantage of being to choose whether they prefer to work part-time or full-time, depending on their individual situation, such as having young children.

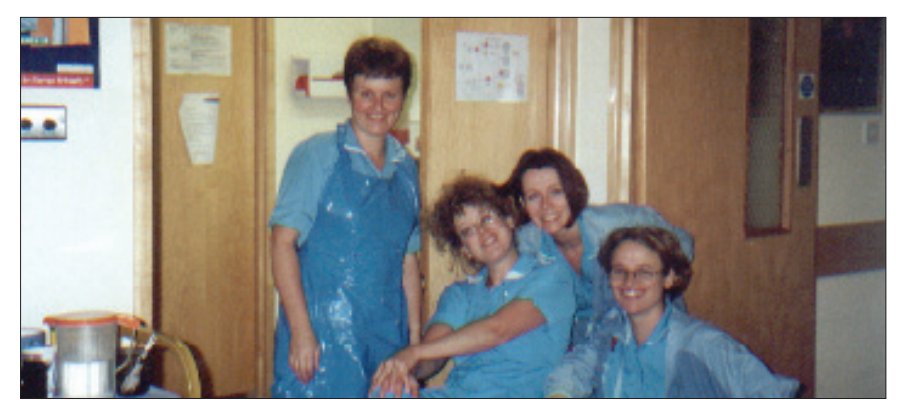

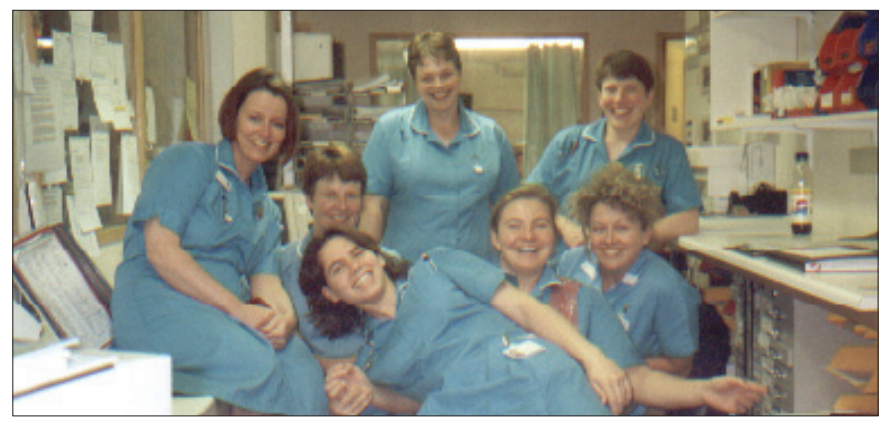

\section{Shifts}

In Spain, the early shift runs from 8 am until 3 pm; the late shift from $3 \mathrm{pm}$ until $10 \mathrm{pm}$; and the night shift from $10 \mathrm{pm}$ until 8 am. Nurses are given a schedule to follow for the whole year. Nurses cannot choose the shift they would like for the next month (as nurses can in England). However, nurses can change shifts with other nurses, or ask for a certain day off.

\section{Work breaks}

Taking breaks depends on the number of staff working and how busy the day is, but usually in Spain, nurses can choose when to take their break or breaks, which are paid breaks, and can decide how long they will be. In England, breaks are non-paid and usually the nursing sister decides the best moment to take breaks, depending on the ward's needs.

\section{PERSONAL COMMENTS}

The above comments outline the main differences we found between ICU nursing in Spain and England. we have tried to be objective describing the situation in both ICUs, but if you would like to know our personal opinion, we think they are just different ways of understanding the nursing world. Of course, we prefer some aspects of English nursing and other aspects of Spanish nursing. For example, due to the lack of professional and economic evolution among Spanish nurses, such as the lack of nursing grades, the 'burnout syndrome' is very common among Spanish nursing staff.

Since in Spain, the ward manager does not supervise the care given by every nurse to their patients, patient care is not always the best, due to lack of knowledge or motivation. However, at the same time, the kind of nursing care given can be more creative and more stimulating, because nurses are free to change something for the better of their patient without needing the permission of the nursing manager. However, one problem of working independently in this way is that a patient's care plan can differ from shift to shift, depending on the criteria of the nurse looking after the patient.

We also feel that the English policy of allowing relatives to stay as long as possible in the ICU is a good one, which is very positive for the patient and their family.

In Spain, we found that having a schedule by which we could predict our shifts for a year was very useful. It meant we could organise ourselves better in Spain than in England, where we only knew our shifts and availability one month in advance.

\section{CONCLUSION}

The best thing for us about our experience was realising that we all have good and bad points, make mistakes, and have different ways of dealing with situations to make us wise. So keep on learning from, and living, new situations! 